\title{
Use of radio-iodinated serum albumin in the study cerebrospinal fluid flow
}

\author{
GORDON BROCKLEHURST ${ }^{1}$ \\ From the Department of Neurological Surgery and Neurology, \\ Addenbrooke's Hospital, Cambridge
}

The evidence which has accumulated over the last 15 years from the use of radio-isotopes in the investigation of cerebrospinal fluid production, flow, and absorption indicates that, whereas small molecules and ions are exchanged between the cerebrospinal fluid and the systemic circulation at a very high rate, the disappearance of protein from the lateral ventricles is primarily dependent upon a relatively slow flow through the classical pathway to the sites of absorption (Sweet, Brownell, Scholl, Bowsher, Benda, and Stickley, 1954). The disappearance half-time ( $\left.\mathrm{T}_{\frac{1}{2}}\right)$ of $\mathrm{I}^{131}$ albumin from the lateral ventricles is grossly prolonged in hydrocephalus, and this observation has been used more recently as the basis of a quantitative method for the diagnosis of this condition (Atkinson and Foltz, 1962; Atwell and Scott, 1966).

The movement of protein through the cerebrospinal fluid pathway can be studied graphically, using a scintillation counter and serial scanning to detect the sites of maximal gamma emission after the injection of radio-iodinated serum albumin into the ventricles (Di Chiro, 1964) - a technique known as RISA ventriculography. By this method the bulk movement of the labelled protein is clearly demonstrated and, in the normal adult, the RISA reaches the basal cisterns in $1 \mathrm{hr}$, the Sylvian fissure in $3 \mathrm{hr}$, the cerebral convexity in $12 \mathrm{hr}$, and by $24 \mathrm{hr}$ the remaining emission is alongside the sagittal sinus (Di Chiro, 1966).

This communication is a preliminary report on the use of RISA in the investigation of various conditions with which alterations in the cerebrospinal fluid dynamics are associated. The purpose of the investigations was, firstly, to assess the reliability and place of certain procedures using this radioisotope in the diagnosis of hydrocephalus, in order to plan surgical treatment, particularly in infants, and, secondly, to see if these methods would give a fuller picture of the behaviour of cerebrospinal fluid in various pathological conditions.

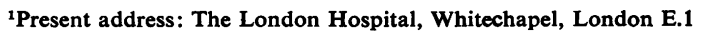

\section{MATERIAL AND METHODS}

Twenty-nine patients have been investigated; in Table $\underset{\mathscr{S}}{\overparen{S}}$ they are arranged according to diagnosis, and it can bo seen that the majority are infants or children with con? genital malformations of the central nervous systemp

TABLE I

RESULTS OF ISOTOPE STUDIES ON CEREBROSPINAL FLUID FLOW

Type of patient Normal Abnormal Abnormal $\dot{\omega}$

\begin{tabular}{|c|c|c|c|}
\hline - & flow & $A$ & $B$ \\
\hline Early myelomeningocoele & 1 & 6 & 2 \\
\hline Late myelomeningocoele & $\mathbf{0}$ & 3 & 4 \\
\hline Congenital hydrocephalus & $\mathbf{0}$ & 3 & $\mathbf{0}$ \\
\hline Late congenital & & & \\
\hline hydrocephalus & $\mathbf{0}$ & 2 & 0 \\
\hline Subdural effusions & 2 & $\mathbf{0}$ & 0 \\
\hline Post-meningitic & & & \\
\hline hydrocephalus & $\mathbf{0}$ & 1 & $\mathbf{0}$ \\
\hline Adult tumours & $\mathbf{0}$ & 2 & $\mathbf{0}$ \\
\hline Adult post-meningitic & & & \\
\hline hydrocephalus & $\mathbf{0}$ & 1 & $\mathbf{0}$ \\
\hline Adult aqueduct stenosis & 0 & 2 & 0 \\
\hline Totals & 3 & 20 & 6 \\
\hline
\end{tabular}

The nine 'Early myelomeningocoeles' were infants investigated between one and four weeks after earl surgical closure of the spinal lesions, and represent larger group of patients which is assuming increasing importance in paediatric neurosurgical practice; in these patients it is of considerable advantage to surgica management if hydrocephalus can be diagnosed early simply, and reliably. The three infants with congenit hydrocephalus (not accompanied by spina bifida) were also investigated in the first few weeks of life. The 'Lato myelomeningocoele' and 'Late congenital hydroce phalus' groups contained infants and young childrem with blocked atrioventricular shunts and possibie recurrence of hydrocephalus. The two infants with sub? dural effusions were investigated because of the possi bility of associated hydrocephalus, and one infant with post-meningitic hydrocephalus was also included in the study.

The five adults investigated consisted of two with tumours which had been previously demonstrated to bo obstructing the cerebrospinal fluid pathway at the level of the brain-stem. Two other adults had benign aquedueg stenosis, demonstrated by myodil ventriculograph 
one had increasing ventricular dilatation and deterioration in conscious level following recurrent meningitis. It was of particular interest in this group of five adults that, although there were clinical features suggesting bouts of raised intracranial pressure, and ventricular dilatation was present in all of them, there was none with frank papilloedema, nor was the pressure of the cerebrospinal fluid markedly raised at the time of the investigations.

The patients were prepared by the administration of Lugol's iodine on the evening before the day of investigation, in order to block the take-up of any free $\mathrm{I}^{131}$ by the thyroid gland, and on the morning of investigation most of the infants were sedated. None of the 29 patients suffered any ill effects which could be directly attributed to the investigation.

The isotope used for these investigations was supplied by the Radiochemical Centre, Amersham, England, in doses of $1 \mathrm{mc}$, contained in a stated volume of isotonic solution, with a stated amount of albumin. In order to avoid contamination from the use of one container for a number of investigations, the initial solution was diluted up to $10 \mathrm{ml}$., and separated into sterile containers, $2 \mathrm{ml}$. $(200 \mu \mathrm{c})$ in each one. These containers were stored in a refrigerator and used only once. Fifty microcuries was calculated to give a safe radiation dose in the various circumstances of the investigations, and the amount of the diluted solution required for this dosage of $50 \mu \mathrm{c}$ depended upon the physical radioactive decay of the $\mathrm{I}^{131}$, but the concentration of albumin was calculated to be between 200 and $250 \mathrm{mg} \%$, depending upon the actual quantity which was initially diluted.

Fifty microcurie doses of RISA were injected into the right lateral ventricle, and during the first hour after the injection a total body count and a body profile scan were performed, using a whole body counting chamber. In this technique, the sodium iodide crystal scintillation counters are contained in a large ring, and are situated vertically above and below the patient. The ring moves slowly along the length of the table bearing the patient, and the bottom scintillation counter was used, collimated, for the body profile, but both counters were used, uncollimated, for the total body count. Anatomical landmarks, such as the top of the head, sternal notch, iliac crests, and feet and the movement of the scintillation counter, were all correlated on the scale of a Beckmann Chart Recorder. In the record of the body profile thus produced the deviations from the base-line are proportional to the intensity of emission detected by the crystal. This investigation, which takes about $15 \mathrm{~min}$, was
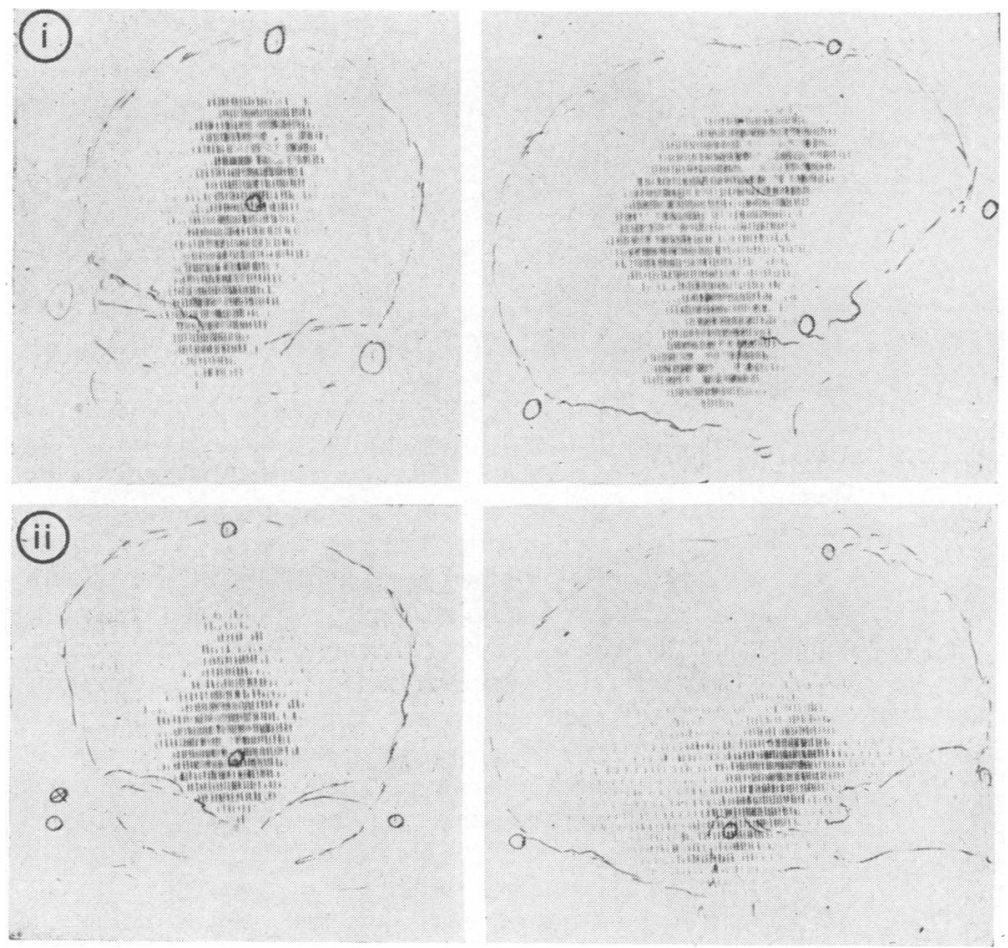

FIG. 1. Normal pattern of isotope ventriculogram. (i) $1 \mathrm{hr}$ after injection. (ii) $6 \mathrm{hr}$ after injection.
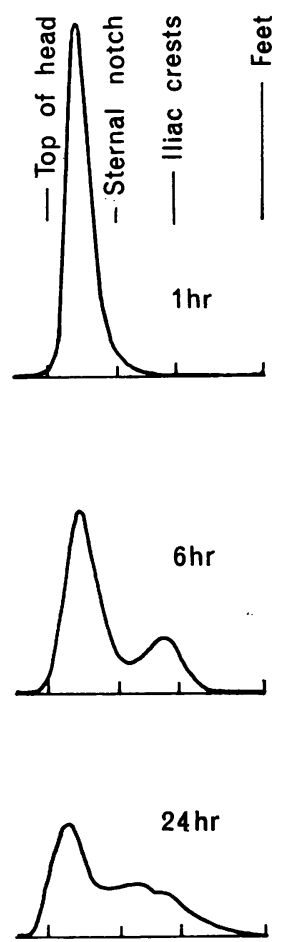

FIG. 2. Normal pattern of profile scan. 

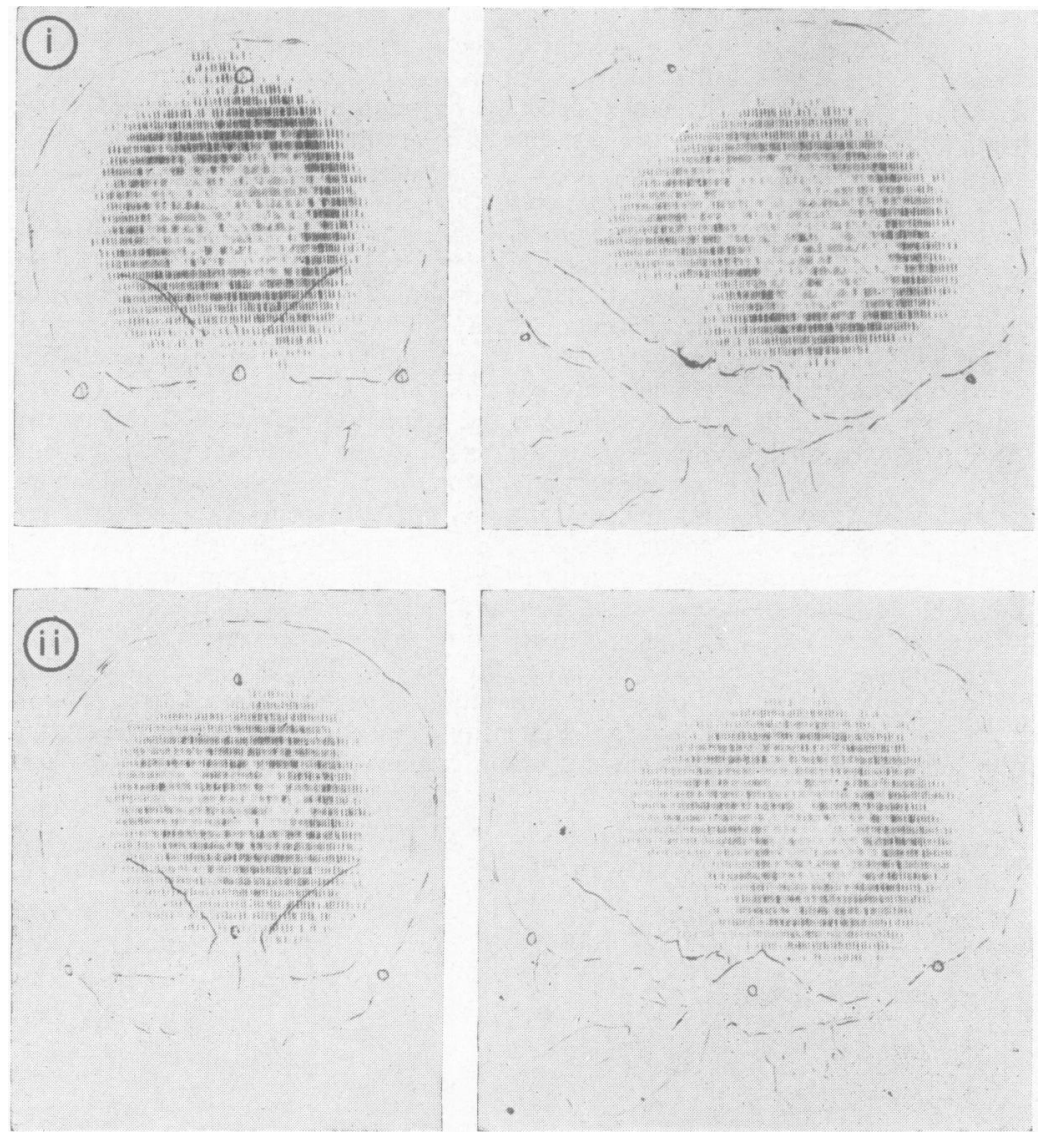

FIG. 3. Abnormal 'A' pattern of isotope ventriculogram. (i) $1 \mathrm{hr}$ after injection. (ii) $24 \mathrm{hr}$ after injection.

repeated just before 6 and $24 \mathrm{hr}$ after the injection.

Isotope ventriculography was also performed at 1 , 6 , and $24 \mathrm{hr}$ after the injection. The ventriculograms were produced as a coloured 'dot scan', using a Picker Magna Scanner. The scintillation counter which traverses to and fro over the head, was collimated, usually with a 19 hole collimator. These scans were performed in the lateral and supine positions, and the colour factor was adjusted to give dark grey dots where the area of high emission tailed off into background activity, and red dots at the centre of the area, where the emission was maximal. In the photographic reproduction of these colour scans for black and white illustrations, the intermediate areas of green and yellow are lost, but must be taken to represent intermediate activity.

\section{RESULTS}

A normal isotope ventriculogram is shown in Fig. 1. The isotope is in the lateral and fourth

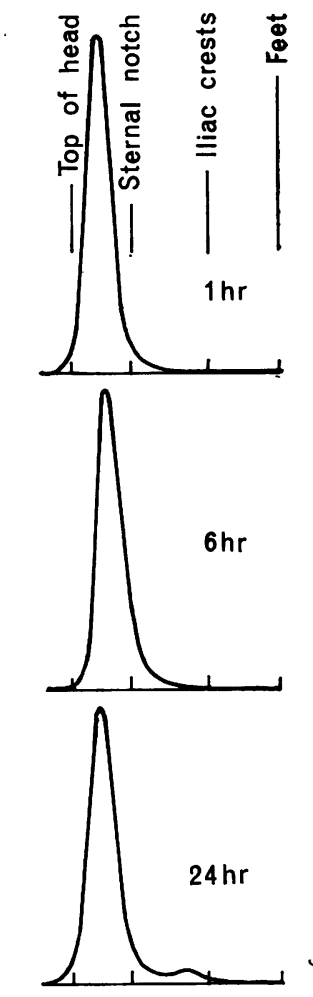

FIG. 4. Abnormal ' $A$ ' pattern of profile scan. ventricles at $1 \mathrm{hr}$, and in the basal cisterns at $6 \mathrm{hr} ; \frac{\mathrm{D}}{2}$ by $24 \mathrm{hr}$ there was no remaining area of high emission in this particular case. The corresponding profile scans, shown in Fig. 2, indicate the appearance of activity outside the head at 6 and $24 \mathrm{hr}$. These results were obtained from a 10-day-old infant with a sacral myelomeningocoele, which had been surgically closed, and with no overt hydrocephalus. Similar results were obtained from the two infants 3 with subdural effusions, but in these, both aged about 6 months, the flow was apparently slightlye slower, and there was a small area of activity left near the sagittal sinus at $24 \mathrm{hr}-\mathrm{a}$ finding similar to $\bigcirc$ Di Chiro's (opus cit.) for the normal adult. It was of incidental interest to find that these two infants with the subdural effusions were the only patients inv whom the total body count dropped significantly over the $24 \mathrm{hr}$ (to $75 \%$ and $50 \%$ respectively). This 0 suggested that in the 6-month-old infants the isotopec 
which reached the systemic circulation after a normal flow through the cerebrospinal fluid pathway was partly metabolized and excreted by $24 \mathrm{hr}$ after the injection into the lateral ventricles, whereas the neonatal infant with the normal flow did not appear to have this capacity. None of these three infants, who were listed under the heading of 'Normal flow' in Table $I$, have shown any evidence of hydrocephalus during the subsequent six months of observation.

The type of flow designated 'Abnormal $A$ ' is illustrated by the ventriculograms in Fig. 3, where the isotope can be seen to have remained in the lateral ventricles during the $24 \mathrm{hr}$. The corresponding profile scans are shown in Fig. 4, and there is only a slight escape of isotope into the body during the same period. The infant with postmeningitic hydrocephalus had a similar profile scan, but the ventriculogram showed the isotope to be in the basal cisterns at $1 \mathrm{hr}$. There was no further movement of the RISA in the later ventriculograms, and the demonstration of the basal cisterns corresponded well with the appearance of the lumbar air study in this infant. Similarly, one of the adult cases, with a recurrent clivus meningioma, showed the isotope to be in the region of the posterior fossa decompression at $1 \mathrm{hr}$, and to be filling the cerebellar and ambient cisterns on the side opposite to that from which the tumour had been approached some two years previously. The later ventriculograms showed no further movement of the RISA, and in this patient there was again a close correlation between the anatomical features of the isotope ventriculograms and those obtained by contrast ventriculography and encephalography. Both of these last mentioned patients have been included under the 'Abnormal A' heading in Table $I$.

All of the patients showing the 'Abnormal A' pattern have also shown other evidence of hydrocephalus. In the neonatal or young infants the evidence consisted of abnormal skull expansion, increased fontanelle tension, or cerebrospinal fluid leaks from the myelomeningocoele wounds; these were all treated by ventriculo-atrial shunts, and the ventricular dilatation confirmed at operation. In some of these cases the clinical signs of progressive hydrocephalus occurred some days or weeks after the isotope investigations. The older infants and children with blocked shunts (a situation which may be confirmed quite conveniently at the time of the isotope studies), manifested their hydrocephalus by such obvious features as suture diastasis on the radiographs of the skull, and raised pressure on tapping the ventricles, or, more subtly, by irritability and mental retardation, accompanied by recognizable ventricular dilatation on the isotope ventriculograms. The response to revision of the ventriculo-atrial shunts was satisfactory, and the parents frequently reported a return of mental alertness.

The five adult patients all showed the 'Abnormal A' pattern and their clinical features and some radiological findings have already been mentioned. Further observations on the two patients with tumours were precluded by the death of one, and the effects of subsequent radiotherapy in the other. The remaining three have shown a good response to shunt procedures.

The 'Abnormal B' type of flow pattern is shown in Figs. 5 and 6 . The ventriculogram shows no flow over $24 \mathrm{hr}$, through the classical cerebrospinal fluid pathway, but the profile scan, illustrated in Fig. 6, shows the isotope to be present outside the head in a significant quantity. Four of the six patients tabulated under this heading showed other evidence of hydrocephalus, and were clinically indistinguishable from the 'Abnormal A' group, and these four all received surgical treatment. Two, however, showed no definite evidence of hydrocephalus, and both of these were children with blocked shunts. Careful examination of the isotope ventriculograms in these two showed that the isotope had, in fact, reached the cerebral surface by $24 \mathrm{hr}$, but not through any recognizable passage through the classical cerebrospinal fluid pathway. In one the isotope was identified to be in the region of the third ventricle at $1 \mathrm{hr}$, and to be over the cerebral convexity by $24 \mathrm{hr}$, and the other showed isotope to be over the cerebral convexity by $1 \mathrm{hr}$ after the insertion into the lateral ventricles. It was considered that these patterns might indicate 'spontaneous arrest' of the hydrocephalus by the formation of abnormal communications between the ventricular system and the subarachnoid space over the cerebral hemispheres. The blocked shunts in these two patients were not revised, and their further clinical progress remained satisfactory over a number of months.

Other features were shown in some of the abnormal ventriculograms: an asymmetrical distribution of the isotope between the two lateral ventricles, as shown in Fig. 5, was a striking feature in those cases where there had been previous cerebral trauma or infection, and one patient with congenital hydrocephalus showed the isotope to be filling a large midline cyst, at 6 and $24 \mathrm{hr}$.

The distribution of the isotope in vitro was studied by injecting $50 \mu \mathrm{c}$ quantities, diluted as described above, into $100 \mathrm{ml}$. flasks containing distilled water, normal saline, $35 \mathrm{mg} \%$ and $70 \mathrm{mg} \%$ albumin respectively. The flasks were not shaken, and serial scanning demonstrated the isotope to be equally and homogeneously distributed through- 

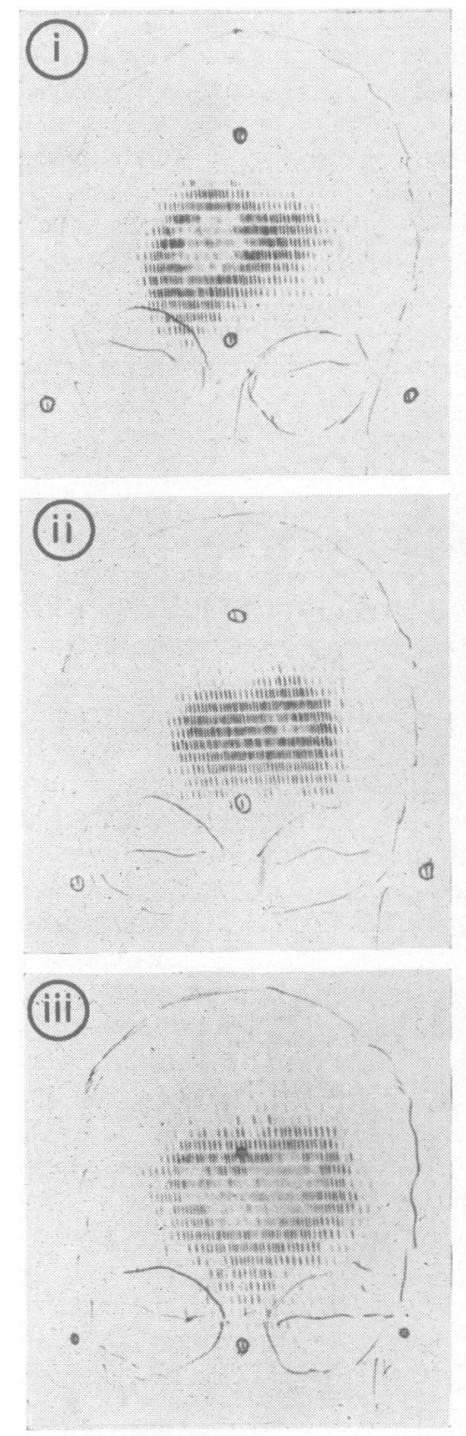
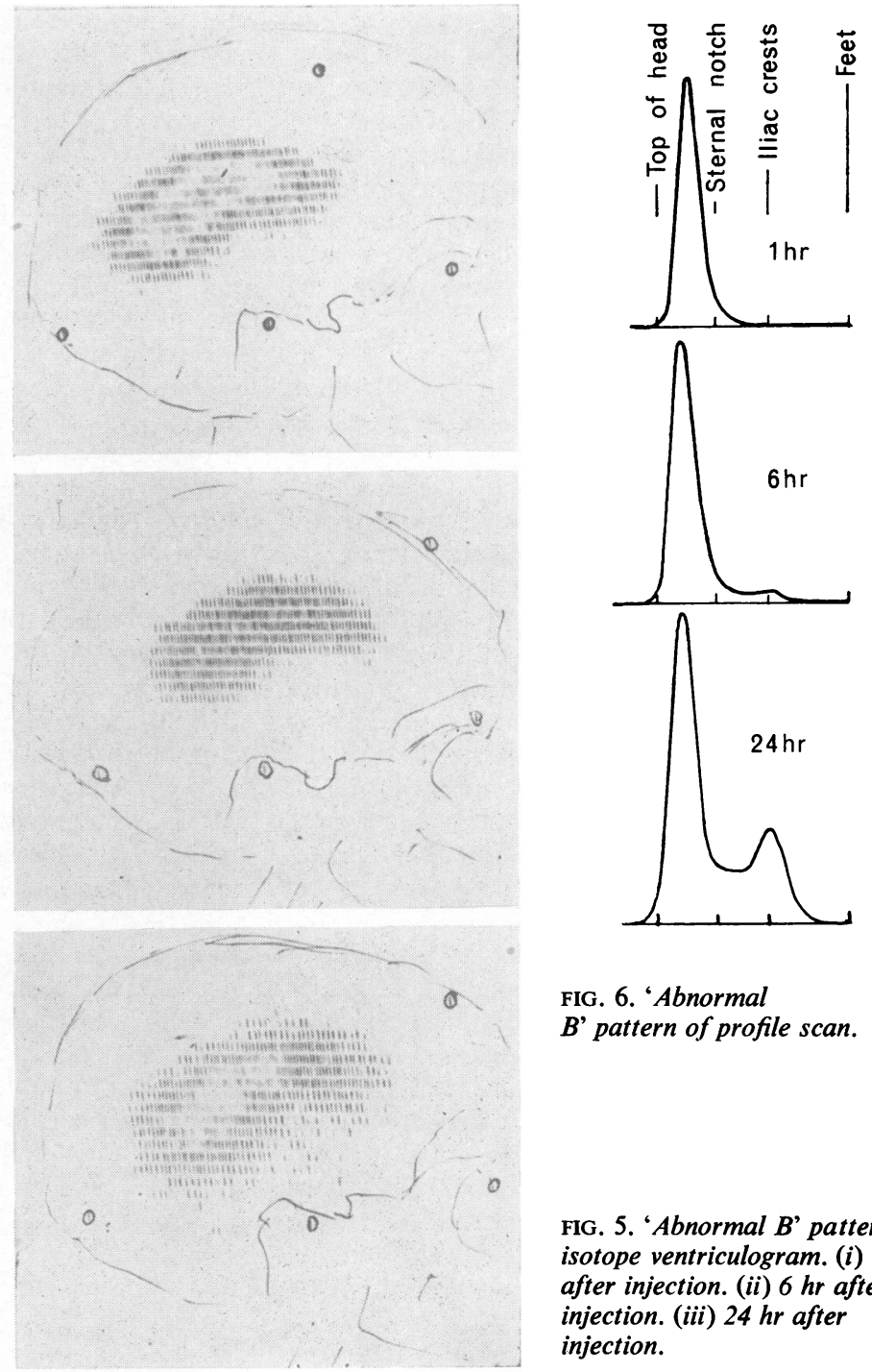

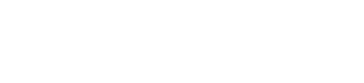

out all of the flasks by half an hour after the injection. The distribution of the isotope in solutions containing a much higher concentration of protein, such as might occur in some pathological situations, has not been studied. No attempt was made to encourage full mixing of the isotope in the ventricles of the patients studied, and the asymmetrical distributions of the isotope occasionally seen appeared to be independent both of the side into which it was injected, and the position of the patient.

The possibility that the isotope which appeared outside the head in the profile scans might in fact be in the spinal canal was excluded both by scanning the spinal column in a number of the patients, when no significantly high activity was found, and also by the observation in the profile scans that the isotope appeared not only over the trunk, but also over the lower limbs and feet.

Quantitative observations upon the appearance of the isotope outside the head in the larger amounts indicated by the 'Abnormal B' profiles have not:so far been made, since repeated ventricular sampling N or repeated venepunctures was undesirable in these young patients. In two of the adults with the 'Abnormal A' pattern, and only a small peak ofw 
activity in the body, repeated venous samples enabled an uptake curve for $\mathrm{I}^{131}$ activity in venous blood to be plotted, and the two curves thus obtained are shown, plotted semilogarithmically, in Fig. 7. It will be seen that there is a significant 'escape' of the isotope into the general circulation in these two patients studied quantitatively. In both of these latter studies a single sample estimation showed that the $\mathbf{I}^{\mathbf{1 3 1}}$ in the plasma by four hours after the intraventricular injection was mostly in a protein bound form.

\section{DISCUSSION}

The isotope ventriculograms performed in these 29 patients suggest that a normal flow, which could be simply defined as 'the appearance of the radioiodinated serum albumin over the upper cerebral convexity by $24 \mathrm{hr}$ after the injection into the lateral ventricles (whether by the classical or some abnormal route)', corresponds with the absence of hydrocephalus, whereas a delay in flow appears sooner or later to be associated with some, or all, of the clinical features of hydrocephalus. The delay in flow is a clearly recognizable phenomenon, and may well precede the clinical manifestations of hydrocephalus by some considerable time. The apparent slowness of the normal flow suggests that the total quantity of protein-containing cerebrospinal fluid which passes through the classical pathway from lateral ventricles to the subarachnoid space alongside the sagittal sinus in $24 \mathrm{hr}$ may be quite small, and that an obstruction to this flow, particularly if incomplete, may result in the slow development of raised intracranial pressure and ventricular dilatation. The relationship of intracranial pressure, ventricular dilatation, and possible variations in the rate of production of cerebrospinal fluid requires further study, but the observations which have so far been made, using these isotope methods, correlate well with long-established clinical observations of the often slow and intermittent nature of progressive hydrocephalus. It remains to be observed whether or not the delayed flow demonstrated in these ventriculograms may be only temporary, and would revert to normal without further treatment. In the absence of papilloedema, or the abnormal skull expansion shown by infants, the confirmation of progressive hydrocephalus by other means is often difficult; the demonstration of patency or obstruction of the cerebrospinal fluid pathway by contrast ventriculography or encephalography is no certain indication of the state of cerebrospinal fluid flow, and ventricular dilatation may be caused by processes other then progressive hydrocephalus. Similarly, raised intracranial pres- sure is dependent upon many factors 'other than hydrocephalus, and it has already been mentioned that hydrocephalus and a normal intracranial pressure may coexist. In many cases the combination of clinical and conventional radiological features enables the diagnosis to be confidently made, but there would appear to be a place for the use of isotope ventriculography in difficult cases, and in patients where the early detection of hydrocephalus might aid surgical management.

On the other hand, the anatomical features of these isotope ventriculograms, although showing a good gross correlation with other radiological methods, lack detailed definition, and cannot replace contrast ventriculography in the accurate delineation of the ventricular system which is required in the diagnosis of tumours.

The body profile scans, and the total body counts, have not so far proved of such diagnostic value with regard to hydrocephalus. The results have raised some questions concerning the behaviour of protein in the cerebrospinal fluid, particularly in various pathological circumstances associated with abnormal isotope ventriculograms. The work of Sweet and his colleagues (Sweet et al., 1954) demonstrated the rapid disappearance of water and electrolytes from the ventricular system, even when there is apparently a total obstruction to the passage of a

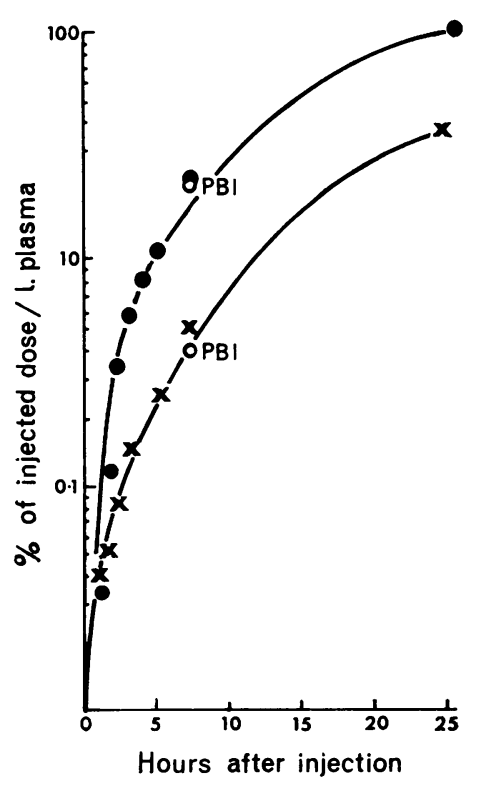

FIG. 7. Uptake curves of $I^{131}$ from two adults with 'Abnormal A' patterns of flow. 
particulate dye, such as indigo carmine. The demonstration by these workers that, in the same circumstances, there was a slow, but definitely measurable, disappearance of $\mathrm{I}^{131}$ albumin from the ventricles, is confirmed by the quantitative observations shown in Fig. 7. The profile scans in the 'Abnormal B' group suggest that, in certain pathological circumstances, the escape of protein directly from the ventricular system may be even greater. This could possibly be associated with a pathological disturbance in whatever factors normally govern the level of protein in the cerebrospinal fluid.

\section{CONCLUSION}

The use of radioiodinated serum albumin in the study of cerebrospinal fluid flow in 29 patients has shown that isotope ventriculography, profile scans, and total body counts can be performed with minimal disturbance of the patients. The isotope ventriculograms have provided a reliable indication of cerebrospinal fluid flow, and abnormalities have corresponded well with other features of hydrocephalus. This method of serial isotope ventriculography would appear to have a place in the diagnosis of hydrocephalus on a reasonably dynamic basis. The profile scans and total body counts have added some information on the complex behaviour of cerebrospinal fluid in pathological conditions. The further extension of these studies to other clinical conditions involving abnormalities of cerebrospinal fluid dynamics would seem worthwhile, and theô̄ value of the methods described in this communi- $Z$ cation and their limitations, could be explored.

These studies were conducted while the author was an Elmore Student in the Department of Medicine, Univer-6 sity of Cambridge, and in receipt of a grant from the $D$ Society for Research into Poliomyelitis and Other Crippling Diseases. The patients were under the care of Mr. Walpole Lewin and Mr. J. R. W. Gleave, from whom much advice and help was received. The isotope tech-? niques were used in the Radioisotope Unit, Addenbrooke's $\vec{\Rightarrow}$ Hospital, with the help and advice of Mr. K. F. Szaz, $\stackrel{9}{+}$ physicist, and the cooperation of all the staff of thato department. The author would also like to acknowledge흠 the help and advice received from Dr. T. D. Hawkins, $\frac{\bar{c}}{6}$ consultant neuroradiologist.

This work has been carried out as part of the require- $\stackrel{D}{0}$ ment of the degree of M.Chir, of the University of $\tilde{w}$, Cambridge.

\section{REFERENCES}

Atkinson, J. R., and Foltz, E. L. (1962). Intraventricular 'RISA' as a diagnostic aid in pre and post operative hydrocephalus. J. Neurosurg., 19, 159-66.

Atwell, J. D., and Scott, P. B. (1966). Cerebrospinal fluid dynamics Th the Arnold-Chiari malformation. Arch. Dis. Child., 41, 172-1

Di Chiro, G. (1964). Movement of the cerebrospinal fluid in humgn $\vec{N}$ beings. Nature (Lond.), 204, 290-291. (1966). Observations on the circulation

Sweet, W. H., Brownell, G. L., Scholl, J. A., Bowsher, D. R., Benta, P., and Stickley, E. E. (1954). The formation, flow and absorp tion of cerebrospinal fluid; newer concepts based on studifs with isotopes. Res. Publ. Ass. nerv. ment. Dis., 34, 101-159- 